\title{
Exploring the Effects of Fermented Chitin Nanowhiskers on Tensile and Thermal Properties of Poly(ethylene glycol) modified Polylactic Acid Nanocomposites
}

\author{
Syazeven Effatin Azma Mohd Asria , Zainoha Zakariaa, Azman Hassan ${ }^{\text {b, }}{ }^{,}$, \\ Mohamad Haafiz Mohamad Kassim ${ }^{c}$, Reza Arjmandid \\ ${ }^{a}$ Department of Chemistry, Faculty of Science, Universiti Teknologi Malaysia, 81310 \\ UTM Johor Bahru, Johor, Malaysia; ${ }^{b}$ Department of Bioprocess and Polymer \\ Engineering, School of of Chemical and Energy Engineering, Faculty of Engineering, \\ Universiti Teknologi Malaysia, 81310 UTM Johor Bahru, Johor, Malaysia; 'School of \\ Industrial Technology, Universiti Sains Malaysia, 11800 USM Penang, Malaysia; \\ dPolymer Processing and Performance Research Group, School of Engineering and \\ Applied Science, Aston University, Aston Triangle, Birmingham, B4 7ET, United \\ Kingdom
}

Abstract The incorporation of fermented chitin nanowhiskers (FCHW) into poly(lactic acid) (PLA) increased the tensile modulus and strength of PLA at the expense of ductility. The brittleness of PLA can be overcome with the use of plasticizer such as polyethylene glycols $(P E G)$. The objective of this study is to investigate the effect of FCHW on the tensile and thermal properties PLA incorporated with PEG as plasticizer (PLA/PEG). PLA/PEG and FCHW reinforced PLA/PEG nanocomposites were prepared using solution mixing technique. Thermogravimetric analysis (TGA) was used to determine the thermal properties while tensile properties were determined from the tensile test. The incorporation of PEG successfully increased the ductility and tensile strength of PLA at the expense of modulus. Based on the tensile properties, $5 \mathrm{phr}$ PEG was chosen for further investigation on the effect of FCHW on PEG modified PLA. Incorporation of $1 \mathrm{phr}$ FCHW PLA/PEG increased the tensile strength and Young's modulus. However, the tensile strength decreased with further addition of FCHW. The elongation at break of PLA/PEG decreased drastically with the incorporation of $1 \mathrm{phr} F \mathrm{CHW}$ and decreased gradually with further increase of FCHW. The thermal stability from TGA of FCHW reinforced PLA/PEG nanocomposites at $5 \mathrm{phr} F \mathrm{CHW}$ content was observed to be significantly higher compared to PLA/PEG, as indicated by $T_{20}$ and $T_{\max }$.

Keywords: chitin, nanowhiskers, polylactic acid, poly(ethylene glycol), nanocomposites

\section{Introduction}

Since the beginning of twenty first century, the interest in sustainability has sparked the research to replace non-renewable resources with renewable sources originating from plants (Yu et al., 2006; Maraveas, 2020). The market relevance of packaging materials made of biodegradable plastics has 
increased over the past few years. They are primarily used as alternatives for conventional plastics such as polyethylene (PE), polypropylene (PP), polystyrene (PS) and polyethylene terephthalate (PET). Among the most studied polymer as a replacement of petrochemical based polymers in packaging industry is polylactic acid (PLA) (Drumright, 2000). PLA is the most promising bio-based and biodegradable thermoplastic, and is considered as a 'green' eco-friendly material. PLA belongs to the family of aliphatic polyesters made up from alpha hydroxyl acids (Garlotta, 2001). PLA is a biopolymer produced by condensation polymerisation of lactic acid, which is extracted from fully renewable resources such as corn, sugar beet or rice. In the packaging field, PLA is used for food trays, water bottles and flexible packaging (Mathew et al., 2005; Petersson et al., 2007). Properties that make PLA a good food packaging material are their high molecular weight, water solubility resistance, good processability and biodegradability (Drumright, 2000).

Besides that, PLA is also a popular material in several biomedical applications (Singhvi, 2019). Research on utilisation of PLA in automotive industries has also intensified (Jung et al., 2011; Notta-Cuvier et al, 2014). PLA exhibits similar tensile strength and modulus, flavour and odour barrier of PET; the temperature stability and process ability of PS; and the printability and grease resistance of PE. Due to these advantages, PLA has been the preferred biopolymer to replace fossil-based polymers. Under industrial composting conditions PLA will degrade into carbon dioxide and water in approximately six weeks (Haider et al., 2018). This is one of the many advantages of PLA when compared to petroleum based polymers such as PET. However, PLA have several weakness such as brittleness and poor thermal stability (Zakaria et al., 2013; Arjmandi et al., 2015). The inherent brittleness of PLA is a major challenge for its use in various applications. Although PLA's stiffness is similar to that of PET, PLA is more brittle with percentage elongation of break to be around $10 \%$ (Ali, 2017).

Chitin is one of the most abundant natural polysaccharides that exist in nature and is found in the outer skeleton of crustaceans such as shrimp, lobster and crab. It is known to possess many desirable properties such as biocompatible, antibacterial (Rinaudo, 2006) and is found to have desirable mechanical properties due to their natural stacks of chitin nanowhiskers (CHW) (Mincea et al., 2012). It exists as a composite with calcium carbonate and protein and forms a strong waterproof material that forms protective outer shells of many crustaceans. It consists of both crystalline and amorphous regions in which the amorphous region can be removed through acid hydrolysis process. However, the organized crystalline region of chitin will remain intact and producing rod like whiskers (Zeng et al., 2012). Chitin nanowhiskers have excellent mechanical properties including high Young's modulus and high fracture strength due to their extended crystalline structure. This will enable the chitin nanowhiskers to be used as reinforcement in nanocomposites (Nishino et al., 1999; Vincent \& Wegst, 2004).

In addition, the reinforcement of PLA using natural nano-fillers is interesting to be considered as it is expected to improve biodegradability besides enhancing the mechanical and thermal properties due to their nanosize crystals (Haafiz, 2016). Rizvi et al. (2011) reported the earliest work on the use of chitin nanowhiskersto reinforce PLA. The chitin nanowhiskers were produced by an acid-hydrolysis technique and melt blending was used to incorporate into PLA. Chitin nanowhiskers were found to decrease the thermal stability of the composites. The stiffness of the composites was found to increase with increasing chitin content while the strength was found to decrease. In a very recent paper, Mohd Asri et al. (2020) investigated the effect of chitin source and content on properties of chitin nanowhiskers PLA nanocomposites. They investigated the use of chitin nanowhiskers from different chitin sources; commercial chitin and fermented chitin whereby fermented chitin was obtained from fermentation of prawn waste. The advantages $\mathrm{CHW}$ from fermented chitin has been previously discussed (Mohd Asri et al., 2020). Tensile strength for PLA/fermented chitin nanowhiskers increased with increasing filler content until it reached optimum values. Young's modulus for the nanocomposites increased with increasing filler content but elongation at break decreased significantly with increasing filler content for all types of nanocomposites. From the thermogravimetric analysis (TGA) results it concluded that the incorporation of fermented chitin nanocomposites increased the thermal stability of PLA.

Many studies have reported the application of polyethylene glycols (PEG) as a plasticizing agent for PLA (Chieng et al., 2013, 2014; Choi et al., 2013; Mohapatra et al., 2014; Ozkoc et al., 2009; Pillin et al., 2006; Sharma et al., 2019; Sheth et al., 1996; Shi et al., 2015; Sungsanit et al., 2012). Plasticizers are organic substances of low volatility that are added to plastic compounds to improve their flexibility, extensibility, and processability. Plasticizers increase flow and thermoplasticity of plastic materials by decreasing the viscosity of polymer melts, the glass transition temperature $\left(T_{g}\right)$, the melting temperature $\left(T_{m}\right)$, and the elastic modulus of finished products (Fink, 2010). PEG is a thermoplastic crystalline homopolymer with low molecular weight formed by ethylene glycol or ethylene oxide (Castillo 2009; Sheth et al., 1997). PEG is a polyether compound with many applications, from industrial manufacturing to medicine. PEG is also known as polyethylene oxide (PEO) or polyoxyethylene (POE), depending on 
its molecular weight. The structure of PEG is commonly expressed as $\mathrm{H}-\left(\mathrm{O}-\mathrm{CH}_{2}-\mathrm{CH}_{2}\right)_{n}-\mathrm{OH}$.PEG contains terminal hydroxyl group and its molecular weight is in the range of $400-40,000 \mathrm{~g} \mathrm{~mol}^{-1}$. It is soluble in organic solvent like alcohol, acetone and chloroform; however, it is insoluble in hydrocarbons. Also, its glass transition temperature $\left(T_{g}\right)$ can vary from $-40^{\circ} \mathrm{C}$ to $-70^{\circ} \mathrm{C}$ (Sheth et al., 1997).

The reasons for selecting PEG as a plasticizer for PLA are its ability to give a large increase in elongation at break, miscibility with PLA, biodegradability and food contactable application (Pillin et al., 2006). However, the addition of PEG into PLA results in a dramatic loss in tensile strength and tensile modulus. One of the earliest studies on PLA/PEG blends was reported by Sheth et al. (1997). They focused on the effect of PLA/PEG ratios on mechanical properties of the blends and the results revealed that when PEG amount was lower than 50\%, the blend had higher elongation and lower modulus. Sungsanit (2012) investigated the effect of PEG with molecular weight of $1000 \mathrm{~g}$ per mol in various PEG concentrations $(0,5,10,15$, and $20 \mathrm{wt} \%)$ on PLA. In relation to plasticizer content, the impact resistance and crystallinity of L-PLA was increased, whereas a decrease in glass transition temperature and lower stiffness was observed. Phase separation has been found in samples which contained PEG greater than 10 wt\%.

In a study of Ozkoc et al. (2009), PLA/PEG films having 0, 3, and 5\% organoclay (Cloisite 30B) were prepared using a lab-compounder connected to a film-casting device. The glass transition temperature $\left(\mathrm{T}_{\mathrm{g}}\right)$ of PLA decreased nearly $30{ }^{\circ} \mathrm{C}$ with the addition of $20 \mathrm{wt} \%$ of PEG. Choi et al. (2013) grafted low molecular weightacrylated-PEG onto PLA via a reactive blending to improve its plasticity. The acrylatedPEG decreased the Young's modulus of PLA by $66 \%$ while increasing the elongation at break of PLA by $380 \%$. Chieng et al. (2013) used low molecular weight PEG (PEG-200) to improve the ductility of PLA. The tensile properties demonstrated that the addition of PEG-200 led to an increase of elongation at break $(>7000 \%)$, but a decrease of both tensile strength and tensile modulus.

Mohapatra et al. (2014) studied the effect of PEG on mechanical properties of PLA and PLA organoclay nanocomposites and found that the addition of organoclay in PLA/PEG matrix improved the tensile and impact properties. Chieng et al. (2014) prepared PLA/PEG/graphene nanocomposites by the melt blending method. In the study, PLA was first plasticized by PEG in order to improve its flexibility and thereby overcome its problem of brittleness. The nanocomposites exhibited a significant improvement in tensile properties at a low graphene loading. The tensile properties demonstrated the addition of $0.3 \mathrm{wt} \%$ of graphene led to an increase of up to $32 \%, 69 \%$ and $21 \%$ in tensile strength, tensile modulus and elongation at break of the nanocomposites respectively, compared to PLA/PEG blend. The nanocomposites also shows enhanced thermal stability compared with PLA/PEG blend in thermogravimetry analysis (TGA). Shi et al. (2015) studied the effect of simultaneous addition of PEG and nanofillers (precipitated calcium carbonate, halloysite nanotubes (HNT)) into the PLA matrix. They found that the nanofillers acted as the reinforcement, while PEG was effective in improving the PLA chain mobility. Sharma et al. (2019) studied the mechanical and thermal properties of PLA-based bionanocomposite films using HNT and PEG by solvent casting process. Addition of PEG increased elongation at break, but reduced the tensile strength. However, addition of HNT in PLA/PEG compensated the fall in tensile strength. Addition of $3 \mathrm{wt} \%$ HNT into PLA/PEG blend containing 5 wt\% PEG increased its elongation at break by $640 \%$ and tensile strength by $22 \%$, while no significant change in Young's modulus was observed. Thermal stability of PLA/PEG/HNT films was higher than those of PLA, PLA/PEG and PLA/HNT films.

To date, no study has been reported on using FCHW as fillers in PEG modified PLA. In the present work, PEG is being used as the plasticizer to reduce the brittleness of PLA and PLA/FCHW nanocomposites. In the preliminary part of the study, the optimum PEG content on PLA was determined. The main objective is to investigate the effect of fermented chitin nanowhiskers (FCHW) content on tensile and thermal properties of PEG modified PLA.

\section{Materials and methods}

\section{Materials}

PLA was obtained from NatureWork ${ }^{T M}$ (PLA 300ID). The density of PLA is $1.25 \mathrm{~g} / \mathrm{cm}^{3}$, melting temperature of $145-155^{\circ} \mathrm{C}$, glass transition temperature of $55-58^{\circ} \mathrm{C}$, crystallinity of up to $37 \%$ and it has an average molecular weight of Mw: $220 \mathrm{kDa}$ and Mn: $101 \mathrm{kDa}$. Chitin sources is fermented chitin (FC) produced by fermentation treatment of tiger prawn waste. The chitinunderwent hydrolysis and dialysis through treatment with hydrochloric acid to produce FCHW (Nair \& Dufresne, 2003). Poly (ethylene glycol) $(\mathrm{Mn}=2000 \mathrm{~g} / \mathrm{mol})$ was purchased from Sigma Aldrich. The solvent used to dissolve the PLA is chloroform, purchased from Merck Malaysia. 


\section{Preparation of PLA and PEG modified PLA}

$10 \mathrm{~g}$ of PLA pellets were fully dissolved in chloroform through constant stirring in a water bath at $60^{\circ} \mathrm{C}$ for approximately 60 minutes according to the method reported earlier (Haafiz et al., 2013). The solution was evenly spread on a glass plate using a fabricated spreader and the film was left to dry by evaporation at ambient temperature for 48 hours. Dried film was then kept in a dessicator prior to analysis. The thickness of the cast film was approximately $100 \pm 0.125 \mu \mathrm{m}$. The PEG modified PLA were prepared by mixing PLA with different PEG contents $(5,10$ and $15 \mathrm{phr})$ following the same method in production of PLA film.

\section{Preparation of PLA/FCHW/PEG nanocomposites}

The PLA/FCHW/PEG nanocomposites were prepared as in previous section by mixing PLA with different FCHW content (1, 2, 3 and $4 \mathrm{phr}$ ). $5 \mathrm{phr}$ of PEG was used based on optimum content obtained from the tensile test.

\section{Field emission scanning electron microscopy}

The study of surface morphology of PLA-PEG films was analysed using Carl Zeiss (Germany) Supra $35 \mathrm{VP}$ microscope using an accelerating voltage in the range $8-10 \mathrm{kV}$. The purpose of this study is to observe the distribution of PEG in PLA/PEG composites. The samples were placed onto specimen stubs and sputter coated with gold prior observation to prevent the accumulation of electrostatic charges on the surface of the samples during electron irradiation. The coated samples were then analyzed using FESEM.

\section{Tensile testing}

Tensile test was performed according to ASTM D882 using L1oyd LRX (USA) machine at ambient condition. Rectangular specimens were cut from the obtained cast film with dimension $60 \times 12.6 \times 0.1$ $\mathrm{mm}^{3}$. The tensile test was performed at crosshead speed of $12.5 \mathrm{~mm} / \mathrm{min}$ with $30 \mathrm{~mm}$ gauge. Tensile strength, elongation at break and Young's modulus were determined. Seven specimens for each formulation were tested and mean value was recorded.

\section{Thermogravimetric Analysis}

Thermogravimetric analyses (TGA) was used to evaluate the thermal stability of the PLA nanocomposites by tracking weight change during a ramp to $600^{\circ} \mathrm{C}$ at $10^{\circ} \mathrm{C} / \mathrm{min}$ with nitrogen purge.

\section{Results and discussion}

\section{Field emission scanning electron microscopy}

Field emission scanning electron microscopy (FESEM) of the PEG modified PLA films were analysed to investigate possible interaction between PEG with PLA. FESEM images of tensile fractured surfaces PLA/PEG at 5, 10 and 15 phr of PEG content are shown in Fig. 1 (a)-(c). The FESEM micrographs of the three samples show characteristics of ductile fractures though not very clear. Considerable voids were clearly observed, which was probably caused by the accumulation of PEG during phase separation. This clearly indicated that the PEG and PLA were not fully miscible to form a homogenous mixture. It was also observed that the voids were uniformly distributed within the matrix. The distribution of the submicron size round shapes increased with increasing PEG content. Besides that, the size of the voids increased with increasing PEG content. The bigger size of the PEG particles of $15 \mathrm{phr}$ compared to 5 phr was most like the reason the slight decrease in tensile strength and elongation at break. Sungsanit et al. (2012) also reported that phase separation occurred and the voids were observed in PLA/PEG blends containing $10 \mathrm{wt} \%$ PEG. From the SEM micrographs of the impact fractured surfaces, they observed evidence of ductile fractures with more and longer fibrils with the increase in PEG content, which were not observed in the current study They also reported that the PEG was equally dispersed in PLA matrix, similar to the current study. 

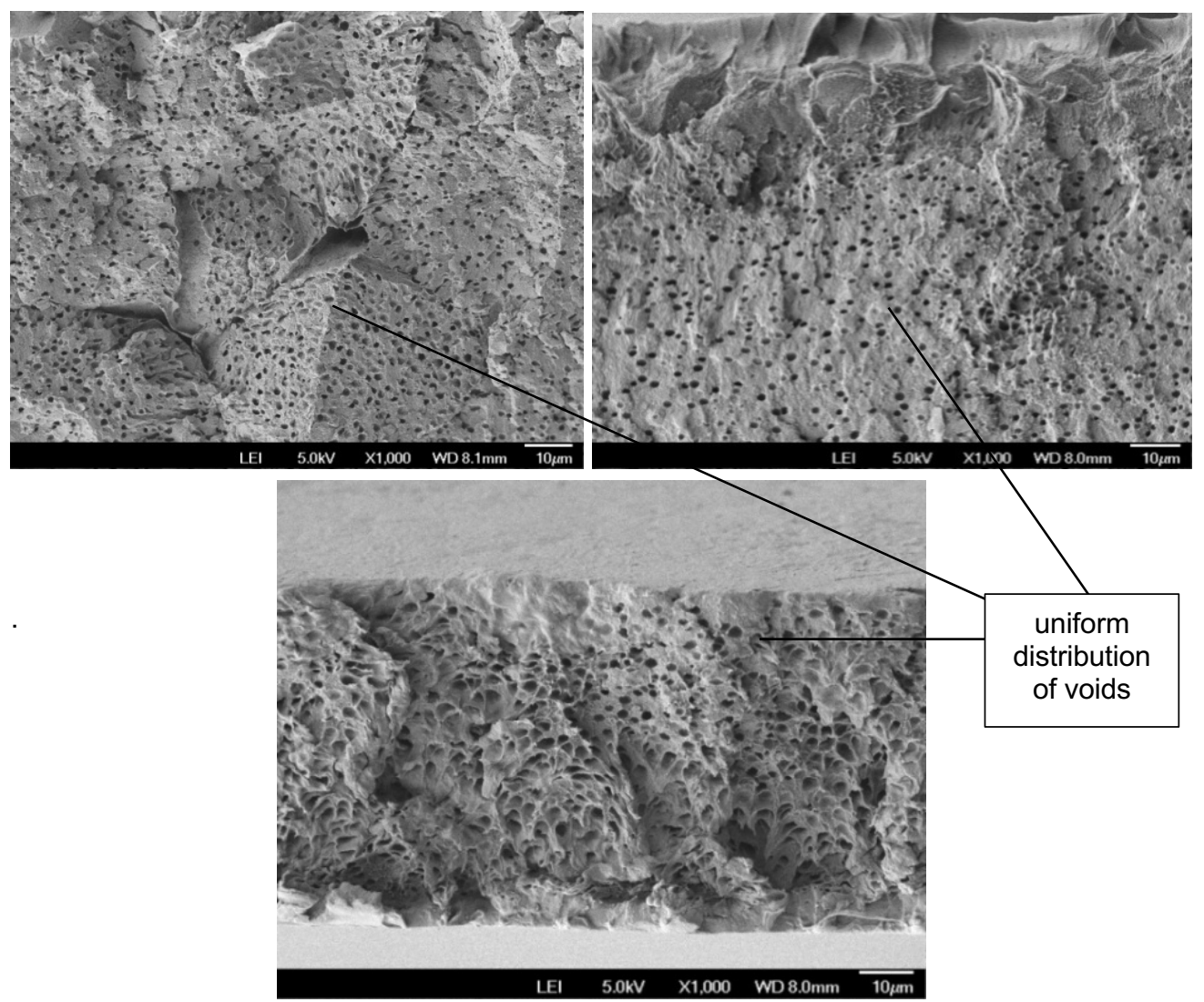

Figure 1. FESEM of a) PLA/PEG5, b) PLA/PEG10 and c) PLA/PEG15

\section{Tensile properties}

The effect of PEG contents on the tensile properties of PLA are shown in Figs. 2-4. As shown in Fig. 2, the tensile strength of PEG modified PLA was found to be higher than neat PLA. The tensile strength increased by $30 \%$ reaching the maximum strength of $17 \mathrm{MPa}$ with the incorporation of $5 \mathrm{phr} P E G$, after which it decreased gradually with increasing PEG content. This is due to PLA and PEG are not fully miscible to form a homogenous mixture as mentioned in previous FESEM section. The possible reason for the higher tensile strength of the PEG modified PLA compared to PLA is that the PEG has strong intermolecular interaction with PLA at low concentration (Lourdin et al., 1997).

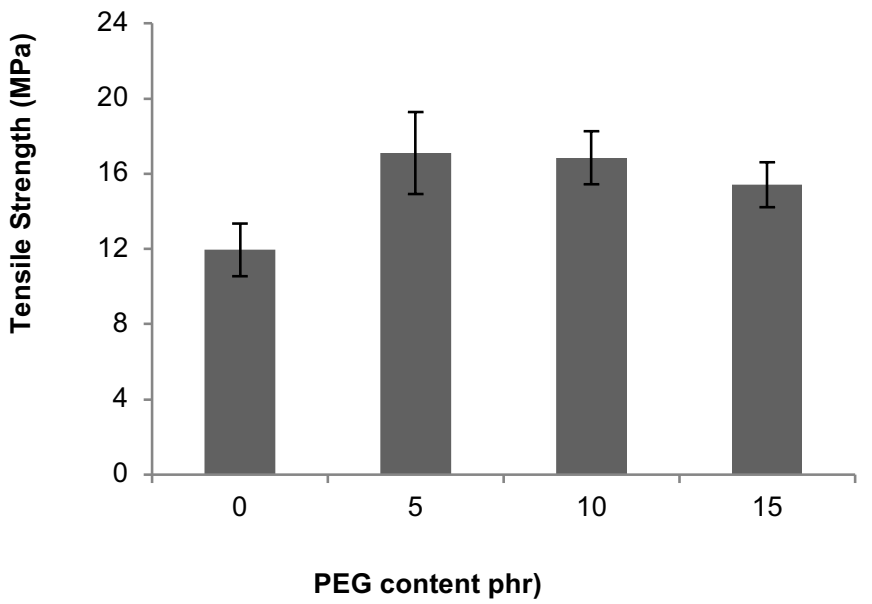

Figure 2. Effect of PEG content on tensile strength of PEG modified PLA 
Fig. 3 shows the effect of PEG content on elongation at break of PLA. It was observed that elongation at break of PLA/PEG samples were higher than neat PLA. All the PLA/PEG samples (5, 10 and 15 phr) showed almost similar values with the highest at $5 \mathrm{phr}$. This is due to immisciblity of PLA and PEG caused voids which due to accumulation of PEG during phase separation. Similar increase in elongation at break of PLA modified by PLA was also observed by other researchers (Courgneau et al., 2011; Chieng et al., 2013). Courgneau et al. (2011) reported that initially at low PEG content (9\%), the increase is relatively marginal. A drastic increase was only observed at $13 \%$ PEG content where the elongation at break of PLA/PEG blend increased by $99 \%$.

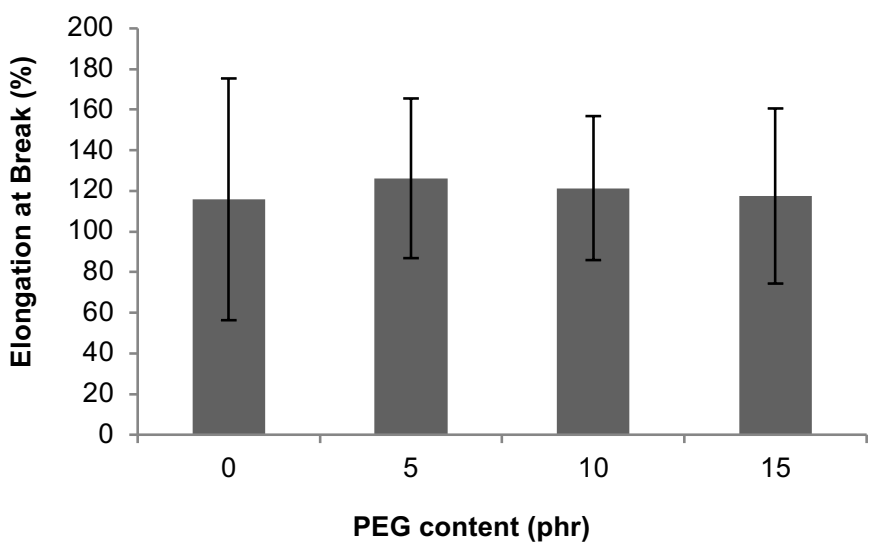

Figure 3. Effect of of PEG content on elongation at break of PEG modified PLA

The effect of PEG content on Young's modulus of PLA/PEG is shown in Fig. 4. The overall results showed that stiffness as indicated by Young's modulus of PLA/PEG are lower than PLA. The incorporation of 5 phr PEG reduced the stiffness of PLA by $70 \%$ and decreased slightly with further increase in PEG content. This is due to the softening effect of PEG. The reduction in modulus with increasing PEG content is consistent with previous studies (Chieng et al., 2013; Choi et al., 2013; Mohapatra et al., 2014). Based on the overall tensile properties, 5 phr PEG was chosen for further study to investigate effect of FCHW content on PLA.

The effects of incorporation of FCHW on the tensile properties of PLA/PEG are shown in Fig. 5. Incorporation of FCHW showed an increase in tensile strength of the PLA/PEG nanocomposites until a maximum values before it decreased gradually due to agglomeration of FCHW. The maximum value (23 MPa) was observed at $1 \mathrm{phr}$. This is an increase of $20 \%$ compared to PEG (5 phr) modified PLA. In our recent study, similar trend was observed for the FCHW reinforced PLA nanocomposites, which the maximum value was also found at 1 phr (Mohd Asri et al., 2020).

As shown in Fig. 6, the elongation at break of PLA/FCHW/PEG decreased significantly with the incorporation of $1 \mathrm{phr}$ of FCHW and decreased gradually with further increase of FCHW. The plausible reason for this decrease is that the FCHW is restricting the segmental chain movement of PLA. Similar trend was observed for the FCHW reinforced PLA nanocomposites whereby the elongation at break decreased significantly with the incorporation of $1 \mathrm{phr}$ of FCHW. However, the elongation at break of FCHW reinforced PLA/PEG at 1 phr FCHW was $30 \%$ higher compared to FCHW reinforced PLA. Thus, it can be concluded that PEG at 5 phr content is effective in enhancing the elongation at break of PLA/FCHW nanocomposites. However, no significant increase in elongation at break at 2 phr FCHW content was observed due to agglomeration of FCHW and restricted segmental chain movement of PLA as discussed above. As shown in Fig. 7, the Young's modulus of PLA/FCHW/PEG5 increased with increasing of FCHW content. The increase was quite apparent at 3 and 4 phr FCHW content. This shows that the modulus is not affected by the agglomeration of FCHW. The increase in the modulus with FCHW content is due to the FCHW restricting the movement of the PLA/PEG matrix. 


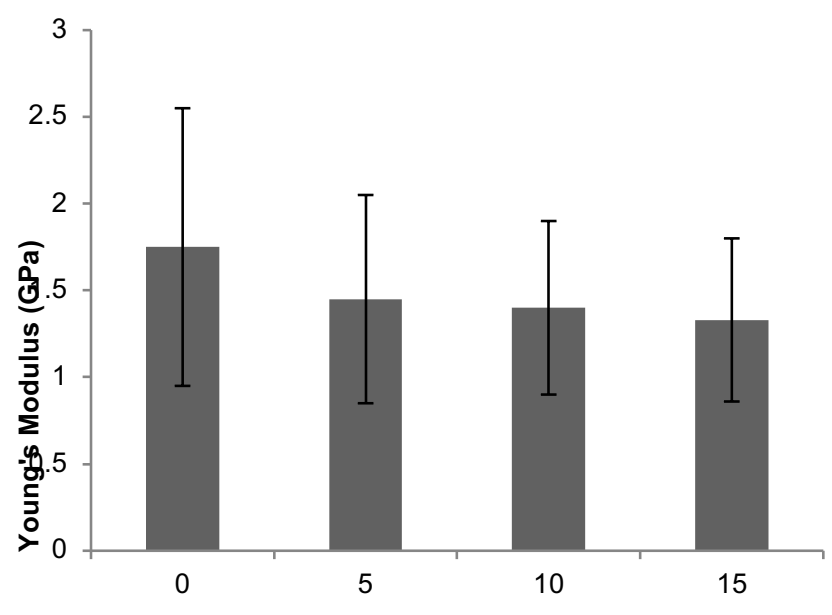

PEG content (phr)

Figure 4. Effect of of PEG content on Young's modulus of PEG modified PLA

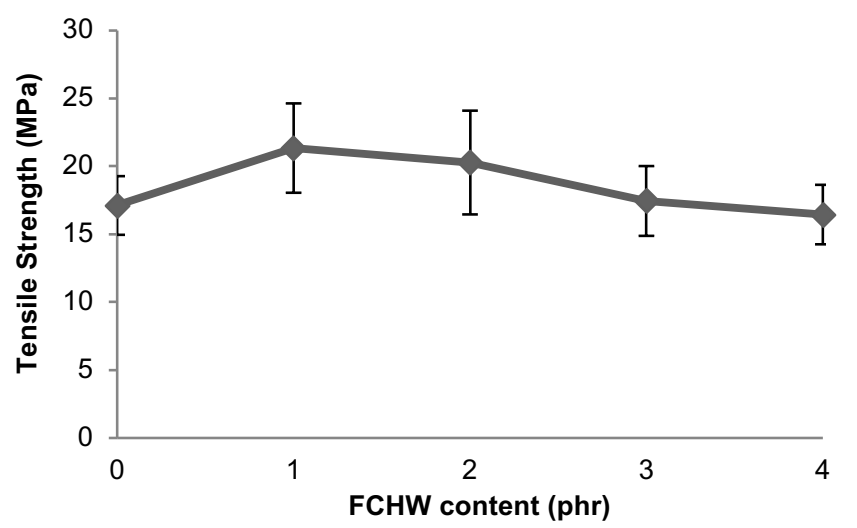

Figure 5. Effect of of FCHW content on tensile strength of PLA/FCHW/PEG5



Figure 6. Effect of of FCHW content on elongation at break of PLA/FCHW/PEG5 


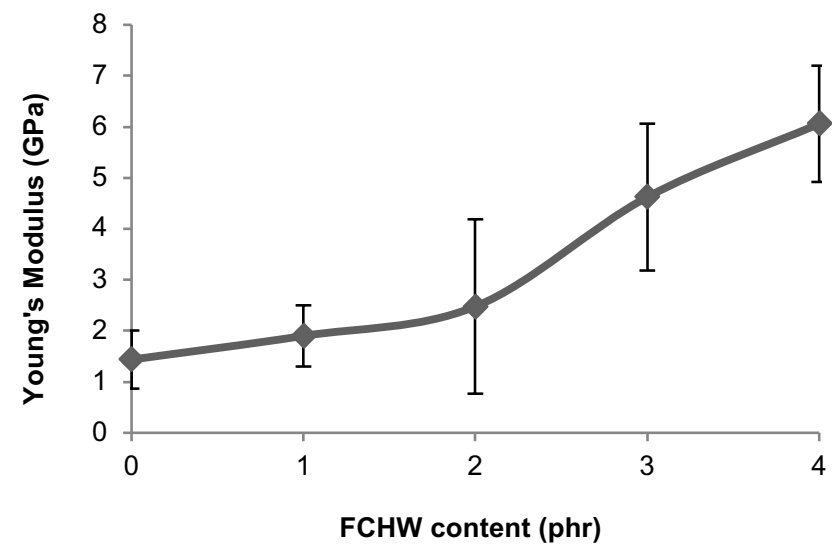

Figure 7. Effect of FCHW content on Young's modulus of PLA/FCHW/PEG5

Thermogravimetric analysis (TGA) curves representing the thermal behaviour of PLA-PEG composites are plotted in Fig. 8. The thermal degradation data $\left(T_{20}\right)$ and peak degradation temperatures $\left(T_{\max }\right)$ obtained from these curves are summarized in Table 1. The TGA and DTG curves of pure PLA and PLAPEG composite showed a similar decomposition pattern of one-step degradation process represented by a single peak.

According to Fig. 8, all samples showed initial weight loss at about $100^{\circ} \mathrm{C}$ to $150^{\circ} \mathrm{C}$. The weight loss was a result of evaporation of moisture. It can be seen that the $T_{20}$ and $T_{\text {max }}$ for PEG modified PLA at all PEG contents were significantly lower than pure PLA which indicates reduction of thermal stability after the addition of PEG. A sharp decrease from 350 to 310 was observed with the incorporation of 5 phr PEG, followed by a more gradual decrease with further addition until $15 \mathrm{phr}$. Similar observation of decreasing in thermal stability with the addition of PEG was also reported by Chieng et al. (2013). They observed higher degree of decrease in $T_{\max }$ when PEG concentration increased from 5 to $10 \%$ as compared 0 to $5 \%$. This result is expected since PEG has lower molecular weight compared to PLA and therefore less thermally stable. The reduction is related to the role of PEG as plasticizer.

The TGA and DTG thermograms of PLA/FCHW/PEG5 is shown in Fig. 9 while their important thermal parameters including $T_{20}$ and $T_{\max }$ are tabulated in Table 2. The TGA and DTG curves of all nanocomposites showed a similar decomposition pattern of one-step degradation process represented by a single peak. The initial weight loss at about $100^{\circ} \mathrm{C}$ to $150^{\circ} \mathrm{C}$ is due to the evaporation of moisture. As the temperature increased, the weight was substantially constant until the temperature associated to the beginning of thermal degradation of the films is reached. The weight losses of the PLA/FCHW/PEG5 nanocomposite films are mainly occurred at the temperature range of $250-500^{\circ} \mathrm{C}$ which is attributed to the breakdown of the polymer chains.

As reported in Table 2, the decomposition temperature of the PLA/FCHW/PEG5 nanocomposites decreased at low content of FCHW. The plausible reason for the initial decrease is that at $1 \mathrm{phr}$ content, the amount of filler is not high enough to cause the oxygen to follow a tortuous path. However, the $T_{20}$ and $T_{\max }$ started to increase at $3 \mathrm{phr}$ FCHW content as compared to PEG modified PLA film. The increase in decomposition temperature indicates the relatively good intermolecular interaction between PLA, PEG and FCHW components through hydrogen bonding. Similar findings were previously reported in the study of effect of incorporation monocrystalline corn straw cellulose and PEG on properties of biodegradable films (Sun et al., 2016). 

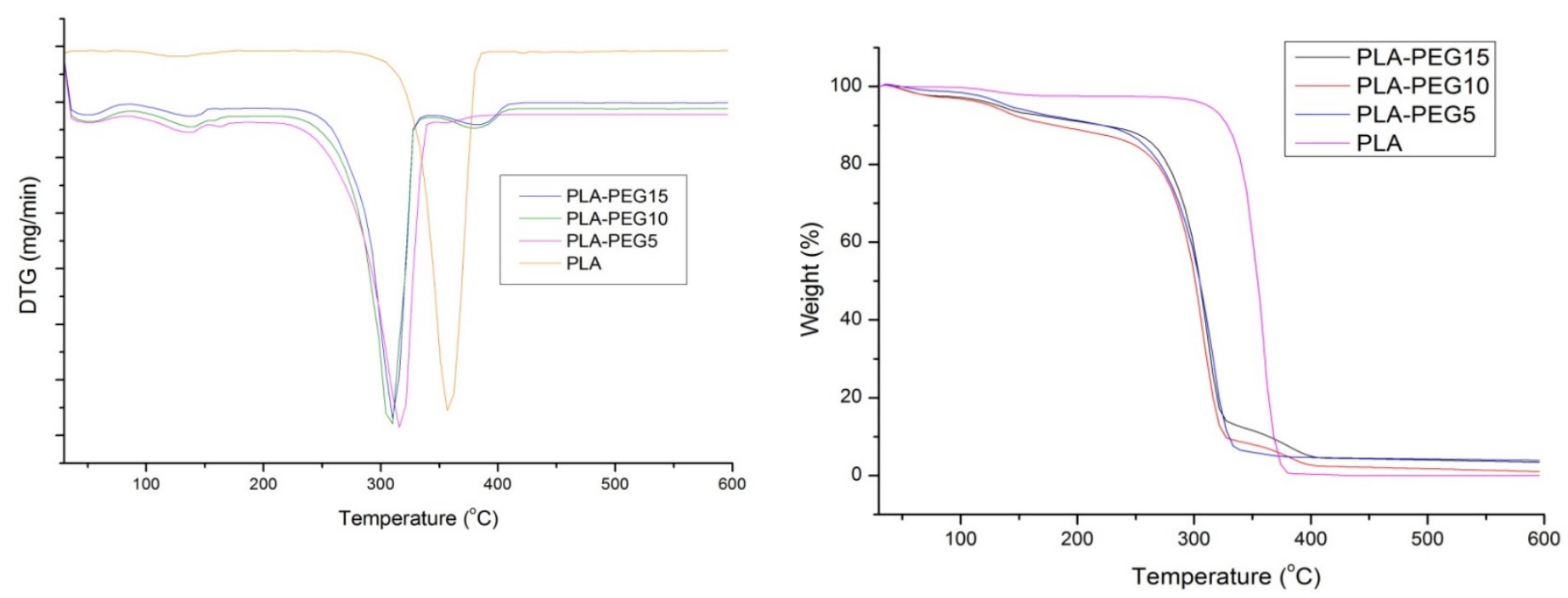

Figure 8. TGA and DTG curves of PLA/PEG composites

Table 1. Degradation temperature for PLA/PEG composite

\begin{tabular}{cccc}
\hline & \multicolumn{2}{c}{$\begin{array}{c}\text { Degradation } \\
\text { temperature }\left({ }^{\circ} \mathbf{C}\right)\end{array}$} & $\begin{array}{c}\text { Residual Weight (\%) at } \\
\mathbf{6 0 0}\end{array}$ \\
\cline { 2 - 3 } & $\mathbf{T}_{\mathbf{2 0}} \mathbf{C}$ & $\mathbf{T}_{\max }$ & \\
\hline PLA & 340 & 358 & 0.03 \\
\hline PLA/PEG5 & 269 & 315 & 3.90 \\
\hline PLA/PEG10 & 269 & 310 & 1.10 \\
\hline PLA/PEG15 & 275 & 310 & 3.44 \\
\hline
\end{tabular}

Besides the intermolecular interaction, there are two other possible reasons for the improvement in the thermal stability for PLA/FCHW/PEG nanocomposites at 3 and 4 phr. Firstly, the thermal improvement could be due to the formation of char which hinders the 'out-diffusion' of the volatile decomposition products, as a direct result of the decrease in permeability. Secondly, the FCHW is retarding the diffusion of oxygen into the polymer matrix by forcing the diffusing molecules to follow a tortuous path. 

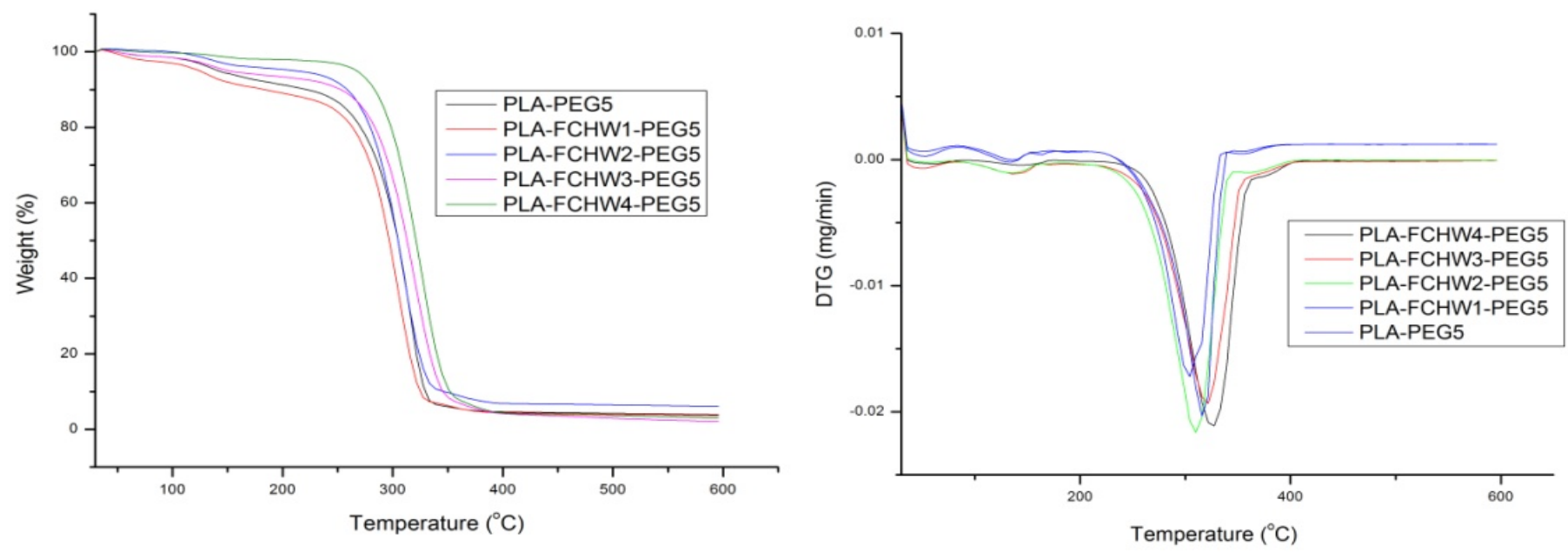

Figure 9. TGA and DTG curve of PLA/FCHW/PEG5

Table 2. Degradation temperature for PLA/FCHW/PEG5 composites

\begin{tabular}{cccc}
\hline \multirow{2}{*}{ Formulation } & \multicolumn{2}{c}{$\begin{array}{c}\text { Degradation temperature } \\
\left({ }^{\circ} \mathrm{C}\right)\end{array}$} & $\begin{array}{c}\text { Residual Weight (\%) } \\
\text { at } 6000^{\circ} \mathrm{C}\end{array}$ \\
\cline { 2 - 3 } & $\mathbf{T}_{\mathbf{2 0}}$ & $\mathbf{T}_{\max }$ & \\
\hline PLA/PEG5 & 269 & 315 & 3.90 \\
\hline PLA/FCHW1/PEG5 & 263 & 304 & 3.74 \\
\hline PLA/FCHW2/PEG5 & 278 & 309 & 6.29 \\
\hline PLA/FCHW3/PEG5 & 283 & 320 & 1.97 \\
\hline PLA/FCHW4/PEG5 & 295 & 325 & 2.75 \\
\hline
\end{tabular}

\section{Conclusions}

The objective of this study was to investigate the effect of FCHW on the tensile and thermal properties of PEG modified PLA. The incorporation of PEG successfully increased the ductility and tensile strength of PLA at the expense of modulus. Based on the tensile properties, 5 phr PEG was chosen for further investigation on the effect of FCHW on PEG modified PLA. FCHW was shown to be effective in increasing the tensile strength and Young's modulus of the PEG modified PLA at the expense of ductility. The elongation at break reduced drastically at $1 \mathrm{phr} F C H W$ content. The thermal stability of PLA/FCHW/PEG nanocomposites was observed to be significantly higher at high FCHW content

\section{Acknowledgments}

The author would like to thank Universiti Teknologi Malaysia (UTM) for the research facilities and Ministry of Higher Education Malaysia for financial support through FRGS Grant 4F377. 


\section{References}

Ali, N. A. 2017. Effect of Polyethylene Terephthalate (PET) on Mechanical and Optical Properties of Polylactic Acid (PLA) for Packaging Application. J. chem. pharm. 9(3), 184-188.

Arjmandi, R., Hassan, A., Haafiz, M. K. M., Zakaria, Z. 2015. Tensile and Morphological Properties of Hybrid Montmorillonite/Microcrystalline Cellulose Filled Polylactic Acid Composites: Effect of Filler Ratio. J. Adv. Mater. Res. 1125, 271-275.

Castillo, R. V., Müller, A. J. 2009. Crystallization and morphology of biodegradable or biostable single and double crystalline block copolymers. Prog. Polym. Sci. 34(6), 516-560.

Chieng, B. W., Ibrahim, N. A., Yunus, W. M. Z. W., Hussein, M. Z., Then, Y. Y., Loo, Y. Y. 2014. Effects of graphene nanoplatelets and reduced graphene oxide on poly(lactic acid) and plasticized poly(lactic acid): A comparative study. Polymers. 6, 2232-2246.

Choi, K. M., Choi, M. C., Han, D. H., Park, T. S., Ha, C. S. 2013. Plasticization of poly (lactic acid)(PLA) through chemical grafting of poly (ethylene glycol)(PEG) via in situ reactive blending. Eur. Polym. J. 49(8), 2356-2364.

Drumright, R. E., Gruber, P. R., Henton, D. E. 2000. Polylactic acid technology. Adv. Mater. 12(23), 18411846.

Fink, J. K. 2010. A concise introduction to additives for thermoplastic polymers. (Vol. 1). John Wiley \& Sons

Garlotta, D. 2001. A literature review of poly (lactic acid). J. Polym. Environ. 9(2), 63-84

Haafiz, M. M., Hassan, A., Khalil, H. A., Fazita, M. N., Islam, M. S., Inuwa, I. M., Marlina, M. M.\& Hussin, M. H. 2016. Exploring the effect of cellulose nanowhiskers isolated from oil palm biomass on polylactic acid properties. Int. J. Biol. Macromol. 85, 370-378.

Haafiz, M. K. M., Hassan, A., Zakaria, Z., Inuwa, I. M., Islam, M. S., Jawaid, M. 2013. Properties of polylactic acid composites reinforced with oil palm biomass microcrystalline cellulose. Carbohydr. Polym. 98(1), $139-145$.

Haider, T. P., Volker, C., Kramm, J., Landfester, K. \& Wurm, F.R. 2018. Plastics of the Future? The Impact of Biodegradable Polymers on the Environment and the Society. Angew. Chem. Int. Ed. 58(1). 50-62

Jung, J. W., Kim, S. H., Kim, S. H., Park, J. K., Lee, W. I. 2011. Research on the development of the properties of PLA composites for automotive interior parts. J. Mater. Sci. 24(3), 1-5.

Lourdin, D., Coignard, L., Bizot, H., Colonna, P. 1997. Influence of equilibrium relative humidity and plasticizer concentration on the water content and glass transition of starch materials. Polymer. 38(21). 5401-5406

Maraveas, C. 2020. Production of sustainable and biodegradable polymers from agricultural waste. Polymers. 12(5), 1127-1148

Mincea, M., Negrulescu, A., Ostafe, V. 2012. Preparation, modification, and applications of chitin nanowhiskers: A review. Rev. Adv. 30, 225-242.

Mohapatra, A. K., Mohanty, S., Nayak, S. K. 2014. Effect of PEG on PLA/PEG blend and its nanocomposites: A study of thermo-mechanical and morphological characterization. Polym. Compos. 35(2), 283-293

Mohd Asri, S. E. A., Zakaria, Z., Hassan, A. Haafiz, M. K. M. 2020. Effect of chitin source and content on properties of chitin nanowhiskers filled polylactic acid composites. IIUM Eng. J. 21(2), 239-255.

Mohd Asri, S. E. A., Zakaria, Z., Hassan, A., Haafiz, M. K. M., Hassan, A., Arjmandi, R. 2017. Isolation and Characterization of Chitin Nanowhiskers from Fermented Tiger Prawn Waste. Chem. Eng. Trans. 56, 139-144.

Nair, K. G., Dufresne, A. 2003. Crab shell chitin whisker reinforced natural rubber nanocomposites. 1. Processing and swelling behavior. Biomacromolecules. 4(3), 657-665.

Nishino, T., Matsui, R., Nakamae, K. 1999. Elastic modulus of the crystalline regions of chitin and chitosan. J. Polym. Sci. Pol. Phys. 37(11), 1191-1196.

Notta-Cuvier, D., Odent, J., Delille, R., Murariu, M., Lauro, F., Raquez, J. M., Dubois, P. 2014. Tailoring polylactide (PLA) properties for automotive applications: Effect of addition of designed additives on main mechanical properties. Polym. Test. 36, 1-9.

Ozkoc, G., Kemaloglu, S. 2009. Morphology, biodegradability, mechanical, and thermal properties of nanocomposite films based on PLA and plasticized PLA. J. Appl. Polym. Sci. 114, 2481-2487

Pillin, I., Montrelay, N., Grohens, Y. 2006. Thermo-mechanical characterization of plasticized PLA: Is the miscibility the only significant factor. Polymer. 47(13), 4676-4682.

Rinaudo, M. 2006. Chitin and chitosan: Properties and applications. Prog. Polym. Sci. 31(7), 603-632.

Rizvi, R., Cochrane, B., Naguib, H., Lee, P. C. 2011. Fabrication and characterization of melt-blended polylactide-chitin composites and their foams. J. Cell. Plast. 47(3), 283-300.

Sharma, S., Singh, A. A., Majumdar, A., Butola, B. S. 2019. Tailoring the mechanical and thermal properties of polylactic acid-based bionanocomposite films using halloysite nanotubes and polyethylene glycol by solvent casting process. J. Mater. Sci. 54. 8971-8983.

Sheth, M., Kumar, R. A., Davé, V., Gross, R. A., McCarthy, S. P. 1997. Biodegradable polymer blends of poly (lactic acid) and poly (ethylene glycol). J. Appl. Polym. Sci. 66(8), 1495-1505.

Shi, X., Zhang, G., Phuong, T. V., Lazzeri, A. 2015. Synergistic effects of nucleating agents and plasticizers on the crystallization behavior of Poly(lactic acid). Molecules. 20(1), 1579-1593.

Singhvi, M. S., Zinjarde, S. S., Gokhale, D. V. 2019. Polylactic acid: synthesis and biomedical applications. J. Appl. Microbiol. 127(6), 1612-1626.

Sun, H., Shao, X., Ma, Z. 2016. Effect of Incorporation Nanocrystalline Corn Straw Cellulose and Polyethylene Glycol on Properties of Biodegradable Films. J. Food Eng. 81(10), 2529-2537 
Sungsanit, K., Kao, N., Bhattacharya, S. N. 2012. Properties of linear poly (lactic acid)/polyethylene glycol blends. Polym. Eng. Sci. 52(1), 108-116.

Vincent, J. F. V, Wegst, U. G. K. 2004. Design and mechanical properties of insect cuticle. Arthropod Struct. Dev. 33, 187-199.

Yu, L., Dean, K., Li, L. 2006. Polymer blends and composites from renewable resources. Prog. Polym. Sci. 31(6), 576-602.

Zakaria, Z., Islam, M. S., Hassan, A., Mohamad Haafiz, M. K., Arjmandi, R., Inuwa, I. M., Hasan, M. 2013. Mechanical Properties and Morphological Characterization of PLA/Chitosan/Epoxidized Natural Rubber Composites. Adv. Mater. Sci. Eng. 2013, 1-7.

Zeng, J.-B., He, Y.-S., Li, S.-L., Wang, Y.-Z. 2012. Chitin whiskers: an overview. Biomacromolecules. $13(1), 1-11$. 